\title{
Claude CALAME, Florence DuPONT, Bernard LORTAT-
} JACOB, Maria MANCA (dir.), La voix actée, pour une nouvelle ethnopoétique

\section{Micheline Lebarbier}

\section{OpenEdition}

\section{Journals}

\section{Édition électronique}

URL : https://journals.openedition.org/clo/856

DOI : $10.4000 /$ clo.856

ISSN : 2266-1816

Éditeur

INALCO

\section{Édition imprimée}

Date de publication : 15 janvier 2010

Pagination : 266-272

ISBN : 978-2-85831-196-5

ISSN : 0396-891X

\section{Référence électronique}

Micheline Lebarbier, "Claude Calame, Florence Dupont, Bernard Lortat-Jacob, Maria Manca (dir.), La voix actée, pour une nouvelle ethnopoétique », Cahiers de littérature orale [En ligne], 67-68 | 2010, mis en ligne le 01 mars 2013, consulté le 01 juillet 2021. URL : http://journals.openedition.org/clo/856 ; DOI : https://doi.org/10.4000/clo.856

Ce document a été généré automatiquement le 1 juillet 2021.

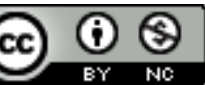

Cahiers de littérature orale est mis à disposition selon les termes de la Licence Creative Commons Attribution - Pas d'Utilisation Commerciale 4.0 International. 


\title{
Claude CALAME, Florence DUPONT, Bernard LORTAT-JACOB, Maria MANCA (dir.), La voix actée, pour une nouvelle ethnopoétique
}

\author{
Micheline Lebarbier
}

\section{RÉFÉRENCE}

Claude CALAmE, Florence DuPONT, Bernard LORTAT-JACOB, Maria MANCA (dir.), La voix actée, pour une nouvelle ethnopoétique, Paris, Éditions Kimé, 2010, 327 p. ISNB :

978-2-84174-531-9.

1 Cet ouvrage qui est une émanation des travaux du GREP (Groupe de Recherche en Ethnopoétique) situe l'ethnopoétique à la croisée des trois disciplines : poésie, musique, littérature. Si l'ethnopoétique englobe toutes les composantes de l'événement parolemusique, se pose la question de la classification délicate entre les différents genres selon les différentes cultures. Le recueil se divise en trois parties. Il brosse un état des lieux, critique parfois, et propose pour certaines contributions de nouvelles méthodes d'analyse.

Dans son introduction Florence Dupont insiste sur les concepts méthodologiques qui sous-tendraient les études d'ethnopoétique. Elle interroge les notions de "genre », de «texte», de «performance», le «contenu de l'énoncé» et le travail de l'ethnopoéticien sur l'énoncé notamment (qu'il soit verbal et/ou musical) qu'il doit réintégrer dans son contexte culturel, sans oublier l'interaction avec l'auditoire. Sans négliger non plus l'arbitraire du choix de l'ethnopoéticien (et de l'ethnologue en général ?) et son implication personnelle dans son objet d'étude. 
3 Cette interrogation se poursuit avec la première partie, "Traversées critiques ", où chaque contribution remet « en cause l'une ou l'autre de ces catégories par l'étude de faits singuliers ».

4 Ainsi Katell Morand examine les réactions contrastées des auditeurs à l'écoute d'un chant à la facture contradictoire. Son analyse, qui s'appuie sur les théories de la psychologie cognitive et des schémas préétablis de la mémoire, ne permet pas de trancher sur l'identification d'un genre ou d'un autre. Chaque genre entraînant pour l'auditeur une réaction et une perception différente.

5 L'étude de Claude Calame se penche sur le "brouillage des règles de genre » à travers les contrastes de formes du dithyrambe ${ }^{1}$ et du péan ${ }^{2}$. Lorsqu'un dithyrambe est adressé à Apollon et un péan à Dionysos, la performance rituelle et la créativité dépendent alors des auteurs de ces chants et les deux divinités se trouvent en final juxtaposées. L'auteur, se fondant sur la structure narrative des deux genres, conclut à la complémentarité culturelle d'Apollon et de Dionysos qui s'exprime à travers "le récit » concernant l'un et le « discours » intégrant l'autre.

6 Ces « Traverses critiques » sont particulièrement bien nommées avec la contribution de Miriam Rovsing Olsen qui se pose la question de la distinction des genres et des critères de classification. En se fondant sur l'exemple du chant dans les traditions orales du Maghreb, elle se propose de réunir les approches littéraire et ethnomusicologique dans son étude. Auparavant, elle pose un regard critique sur les travaux de certains chercheurs : qui sur l'impasse sur la performance, qui sur la confusion des genres, qui sur la confusion des personnages joués par les chanteuses, qui sur l'analyse musicologique... De plus, elle regrette le manque de fiabilité des critères de classification dus au fait que les «concepts de genre et/ou de forme " n'aient pas " nécessairement été définis au départ» (p.63). Et si les critères de classification sont mieux définis, le consensus n'est pas forcément établi entre les différents chercheurs. Elle constate, par ailleurs, le clivage entre les littéraires et les ethnomusicologues et celui de leurs différentes terminologies dépendant de leurs disciplines. Quant aux comparaisons, elles s'avèrent difficiles vu les sens différents donnés aux dénominations des divers inventaires. En conclusion, l'auteur esquisse une nouvelle approche où serait "pris en compte l'ensemble des éléments pertinents de l'acte énonciatif et de la situation d'exécution..." et où serait renouvelée "la compréhension des termes vernaculaires » et des dénominations (p.66). Elle illustre son propos en prenant pour exemple l'analyse de quelques vers d'un rituel de mariage de l'Anti Atlas.

7 Carole Boidin, dans son enquête sur les berceuses au Yémen, se heurte au risque d'agresser la pudeur non seulement de la femme qui chante (près du berceau qui se trouve dans sa chambre, lieu intime) mais aussi celle de la famille. Aussi les berceuses ont-elles été enregistrées hors contexte et étudiées, d'une part comme genre dans leur pratique sociale, et d'autre part comme forme dans leur rythme et leur structure musicale. À partir de deux questions simples : qui chante ? et à qui ?, l'auteur prend en compte la voix, les gestes, la mélodie autant que les paroles, ainsi que le destinataire du chant (l'enfant) autant que le sujet qui chante. Elle examine ensuite les variations existant entre le chant réservé au domaine de l'intime et le chant produit devant et pour un public, ainsi que la position et l'implication du chanteur (de la chanteuse) au sein de la performance. À la lumière de cette analyse est mis au jour le sens de certaines parentés génériques qui sont apparues au cours des enquêtes. Le genre serait alors soumis aux conditions d'énonciation. 
8 Autre questionnement, par Christine Guillebaud, sur le statut de la parole chantée et du chant de possession, à travers un corpus de chants rituels pulluvan («chants de tremblements »), recueilli en Inde du Sud, au Kerala, lors d'un culte domestique dédié aux divinités serpents. Elle aussi constate les sens différents que prend une même énonciation en fonction des lieux et des circonstances. En dissociant les chants de possession hors de leur contexte, l'auteur se propose de les «identifier en entités plus ou moins autonomes»(p.92), et s'interroge sur l'interaction entre la transe des possédées et le chanteur, et sur l'efficacité de ce rituel chanté qui repose sur plusieurs configurations dans sa mise en œuvre.

9 Qu'est-ce qu'un silence musical ? Pour Pierre Letessier, c'est un passage à la «parole sèche ", sans musique. Il ne peut se comprendre "sans la codification qui régit l'arrêt de la musique» (p.110). En rappelant le fonctionnement et les spécificités d'une comédie romaine, il examine les variations de cette codification du silence dans le théatre de Plaute : geste, parole, regard... Convention du silence qui est susceptible de susciter la sortie du personnage, la clôture de la scène et le rire des spectateurs. La fonction rituelle du théâtre dans la Rome antique pose la notion de «variables constantes » qui devraient éviter « l'idée même du modèle » (p. 119). La variation serait nécessaire au sein des constantes du genre théâtral pour provoquer le rire du spectateur et éviter le figement du spectacle.

10 Saut dans le temps et dans l'espace, John Leavitt s'attarde sur l'ethnopoétique nordaméricaine et ses différents courants depuis ses origines avec Boas et son recueil de textes oraux, jusqu'aux travaux de Snyder et de Rothenberg, en passant par ceux de Dell Hymes et de Sapir. Puis il esquisse les perspectives d'une nouvelle ethnopoétique, notamment européenne qui, dépassant «son lien étroit avec la pratique de la poésie », s'inscrit dans une «autre constellation disciplinaire». Celle entre autres de « théoriciens de la littérature ».

11 La deuxième partie de ce livre, «La douleur est une fête ", offre quelques illustrations à la question du genre soulevée par certaines des contributions précédentes. La douleur, la mort, le manque (l'angoisse et la jalousie - autre forme de mort pour l'épouse détrônée - avec l'article de Sandra Bornand) sont exprimés par la voix, la musique, le geste.

12 Le texte de Francis Marmande est un hymne aux auròsts (cris/ lamentation funèbres des femmes lors des funérailles dans la Vallée d'Asp) autant qu'aux femmes qui les proféraient. De cette tranche de vie oubliée dans les Pyrénées-Atlantiques du $\mathrm{XIX}^{\mathrm{e}}$ siècle, l'auteur exhume quelques vestiges d'une tradition orale dont il ne reste que peu de mémoire. Ces femmes et leurs clameurs improvisées, imprécations contre la mort et l'absence, les vivants et leurs compromis, sont comparées aux grandes figures du jazz. On assiste alors au parcours d'un érudit passionné de jazz et amoureux de ce que fut la vie et la voix de ces femmes dans cette vallée des Pyrénées. Hommage à ce qui fut, à ceux (celles) qui furent.

13 Voix de femmes encore avec l'article de Sandra Bornand qui analyse le marcanda, rituel chanté « spécifique aux noces polygames » chez les Zarma (Niger), lors du mariage d'un homme avec une nouvelle épouse. L'arrivée d'une nouvelle femme fait perdre son rang à la «dernière épousée » et donne lieu à ce mercanda où "grandes » et "petites " épouses, s'affrontent sous forme de joutes chantées. L'approche de l'auteur, énonciative et pragmatique, démontre combien ces chants permettent aux femmes dont l'époux se 
marie une fois de plus, d'exprimer leur douleur, leurs craintes et leur impuissance, mais aussi d'entendre la voix de l'ordre social et d'accepter, à la fin, de s'y résigner.

Estelle Amy de la Bretèque met en regard la spécificité de deux types de chants soutenus par deux types de hautbois ; chacun participe de deux répertoires, l'un chante la peine, l'autre la joie. La tristesse des paroles des chants de deuil est renforcée par l'instrument de musique spécifique aux funérailles et par des formules « au sémantisme obscur » mais dont la présence récurrente soutient sinon provoque l'extériorisation de la douleur. Ce répertoire exprimant la tristesse n'est pas réservé qu'aux situations funèbres, il peut aussi dire la peine dans des situations quotidiennes: exil, absence, regrets. En revanche, au répertoire joyeux sont attribués un autre type de hautbois et un autre type de chant. Cette bipolarité se prolonge également autour des fêtes calendaires dans ce que l'auteur appelle joliment « un calendrier des émotions ».

Enquêtes délicates que celles qui ont trait à la mort et à ses rituels, affirme d'emblée Hélène Delaporte, particulièrement en Épire (Grèce). Car "Pleurer est un acte énonciatif (...) Chanter équivaut à pleurer » (p. 197) et par le chant, la mélodie, chanter la mort peut attirer celle-ci, alors que les paroles sans mélodie n'ont pas la même portée. C'est la mélodie qui rend les paroles dangereuses, et le rapport paroles/mélodie est complexe. Les pleurs stylisés, insérés dans les paroles offrent un cadre à la tristesse de la pleureuse. Cadre dont elle sort pour l'exprimer par des formules toutes faites, des cris, des modulations particulières. La tristesse est dans la voix plus que dans les mots et l'analyse démontre que les mêmes paroles peuvent être chant ou lamentations, selon la mélodie. L'auteur pose alors la question de l'efficacité du sémantisme, celui-ci étant paradoxalement présent dans le chant et tronqué dans la lamentation.

Chez les Tsiganes de Ceuaş (Transylvanie) le rituel funéraire, étudié par Filippo Bonini Baraldi, redistribue la place et le rôle de chacun, parent ou étranger, qui déterminent le degré de chagrin qu'il est convenu d'afficher. Le degré de chagrin affiché de la parentèle provoquant le degré de compassion de l'étranger, on assiste à la reconstruction de la parenté du défunt au cours des différents « discours pleurés » et à la mise en relation complexe des membres de la parenté entre eux. L'auteur démontre que ce discours pleuré retisse les relations que le départ du défunt a perturbées.

Enfin la troisième partie de ce recueil, fait ressortir combien le parlé-chanté magnifie l'expression d'une culture au travers d'exemples divers.

18 Ainsi Christiane Seydou analyse l'examen du jeu phonique dans la poésie profane des pasteurs nomades peuls du Mali, où chacun, pendant sa solitude, compose et mémorise un «interminable poème» dont le sujet est le troupeau, les animaux et leurs caractéristiques, et le cadre, la brousse et la saison sèche. À son retour, chacun participera à un concours où seront pris en compte l'entretien du troupeau et la déclamation du poème. La force de l'expression vocale - et de toutes ses composantes est déterminante dans la réception du message. Cependant, il ne s'agit pas d'un récit mais d'une mise en mots qui relève d'une "virtuosité vocale ». Seule importe alors la musique des mots qui entraîne une manipulation voire une épuration de la langue. Le défilé des troupeaux se double d'un défilé de mots. L'auteur replace le sens de cette performance dans son contexte. Un des rares biens tangibles d'une population nomade reste l'expression verbale et le troupeau. Cette manifestation met en scène l'intrication entre l'un et l'autre, sur laquelle repose l'identité peule.

Si la linguistique a découvert tardivement l'expression des affects, l'ethnomusicologie se fonde sur l'approche culturelle de la musique pour l'appréhender. Bernard Lortat- 
Jacob réfute le clivage langue/musique et se penche sur la mise en œuvre des mécanismes du fonctionnement des affects. Les composantes affectives et attributives de la langue et de la musique étant étroitement intriquées leurs polarités peuvent s'inverser. Si « une musique "parle" (...) une langue "chante" » (p. 252). La musique des mots fait suffisamment sens pour que ses registres et modulations soient analysés. L'auteur examine plusieurs cas : registres de tonalité pour le même mot qui exprime cependant des sens différents, interjections sonores qui expriment une variété de sentiments.

La performance orale permet de décoder sens et sentiments différents, mais qu'en est-il du poème écrit? Tristan Mauffrey interroge «ce lien construit entre texte écrit et tradition orale » et recense les traces de la performance chantée dans les odes de la Chine ancienne. Pour ce faire, il se place en tant qu'antiquisant plutôt qu'en sinologue car les odes sur lesquelles il fonde son étude couvrent la période du XIe au VIIe siècle avant notre ère. Il discute les travaux de $\mathrm{M}$. Granet qui interprète les signes d'oralité présents dans le texte sans pour autant que ces signes constituent une trace, puis ensuite les travaux de E. L. Shaughnessy qui, s'appuyant sur plusieurs sources, « élabore des hypothèses sur l'histoire et l'usage de ces textes » (p. 260); enfin ceux de M. Kern qui s'attarde sur la transmission orale des textes, la maîtrise orale du texte rendant seule possible la performance. À partir de ces trois analyses, l'auteur recense l'apport de ces différentes problématiques à la démarche ethnopoéticienne pour l'étude des textes anciens.

21 Maxime Pierre, quant à lui, étudie les termes utilisés autour de la voix dans la Rome antique et leurs sens parfois contradictoires. L'expression de la voix, qu'elle émane d'un humain, d'un animal ou d'un instrument, ou encore de situation où le souffle porte le son, n'incluait pas nécessairement une mélodie ou un sens particulier. Canere, cantare, carmen..., sont des termes qui, parvenant à notre époque, n'ont gardé que quelques traces du sens ancien.

Enfin, saut temporel de l'antiquité, qu'elle soit romaine ou chinoise, à nos jours avec les deux contributions de Jocelyn Bonnerave et Maria Manca pour clore ce volume.

Jocelyn Bonnerave se propose de distinguer le "parler-chanter » - ce qu'il est, ce qu'il n'est pas - du parler ordinaire, et des possibilités offertes par la musique. À travers les portraits de deux « parleurs de jazz » français, elle observe comment le parler-chanter construit un sens et le situe entre les variables du parler ordinaire et du chant artistique. Ces deux figures particulières, pour lesquelles art et vie s'entremêlent, usent d'agencements de mots et de sons qui produisent des « effets de sens singuliers » tout en s'appuyant sur une pratique instrumentale ${ }^{3}$.

Le slam qu'étudie Maria Manca est une autre forme de chanter-parler, qui rassemble un éventail de jeux: jeux de mots, de syntaxe et de sons. La diction et la scansion particulière "produisent une musicalité à la limite du chant»(p.192). Le texte proclamé, claqué porte une dimension violente. Pas de règles, ici seul compte l'effet produit sur l'auditoire (ou bien y aurait-il des règles implicites ?). Le slameur s'expose et l'auditeur-miroir renforce (ou non) le moi du slameur qui se met en scène dans son texte et dans sa performance. L'auteur aborde les différences entre slam et poésie en décrivant ce que le slam n'est pas, mais en définissant le slam comme une «nouvelle sorte d'oralité », qui crée, de façon éphémère, une relation à autrui ; bien que cette relation puisse être régulière. 
25 En refermant ce recueil aux apports variés, on a le sentiment d'avoir abordé de multiples aspects que présente l'ethnopoétique et d'en avoir retiré quelques outils méthodologiques. Toutefois, à travers l'état des lieux critique qu'en ont fait certains auteurs je n'ai, pour ma part, pas toujours pu clairement discerner tous les contours qu'ils souhaitaient tracer de la discipline. Mais l'essentiel n'est-il pas, comme l'exprime Florence Dupont en conclusion de son introduction, d'avoir en approchant « la poésie et la musique des autres » avancé sur le chemin d' "un apprentissage d'humanité » (p. 17) ? Et celui-ci, certes, est complexe.

\section{NOTES}

1. Poème lyrique à la louange de Dionysos.

2. Hymne en l'honneur d'Apollon.

3. Regrettons ici une non-concordance entre les notes et les appels de notes à partir de la note 3 ; et en général que les notes, nombreuses dans plusieurs contributions, soient renvoyées à la fin de chaque article. Des notes infra-paginales auraient rendu la lecture de certains textes plus aisée. D'autre part, signaler le nom de l'auteur (ou le titre de l'article) dans les titres courants aurait permis au lecteur de se repérer plus facilement dans le volume. 